\title{
Die Rückkehr zur Vernunft im Gesundheitswesen des 21. Jahrhunderts
}

Max Geiser

Korrespondenz:

Prof. Dr. med. Max Geiser

Gossetstrasse 49

CH-3084 Wabern
Die Berner Literaturstudie über die Homöopathie brachte keine Überraschung. Albonico und Ferroni $[1,2]$ bezeichnen die im Lancet publizierte Literaturstudie [3] als die derzeit umstrittenste Homöopathiestudie. Nach meiner und der im Lancet-Editorial «The End of Homoeopathy» ausgedrückten Beurteilung brachte die eigentlich überflüssige Literaturstudie keine Überraschung [5], da die Homöopathie längst als Scheinbehandlung und bald nach ihrer Erfindung als Irrtum erkannt worden ist [6-8]. Die Publikation der Studie, des Kommentars dazu [9] und der massiven Kritik des Entwurfs des WHO-Berichtes über die Homöopathie [10] korrigiert reichlich spät den Ausrutscher des Lancet von 1986. Damals wurde eine fälschlicherweise als Doppelblindstudie über eine angeblich wirkungsvolle

\section{Le retour de la raison dans la santé publique du XXIe siècle}

L'article évoque les vaines tentatives de vouloir prouver l'efficacité et le caractère scientifique de I'homéopathie. Les effets secondaires psychiques des placébos induits sous la forme d'une brève influence par suggestion de l'état subjectif grâce à la sécrétion de plusieurs neurotransmetteurs, ne modifient en rien l'inefficacité du placébo contre les suites de maladies et de lésions sérieuses de l'organisme. La recherche de la vérité et, dans la foulée, le progrès dans l'art de guérir, se fondent comme dans tous les domaines de l'activité humaine sur I'identification et l'élimination des erreurs et des dogmes. Les pratiques qui doivent leur survie à la guérison naturelle et à la force régénératrice de l'organisme ainsi qu'à une influence de courte durée exercée sur l'état subjectif du patient et rendue possible par la crédulité et le désespoir, n'améliorent en rien l'efficacité et l'économie du domaine de la santé. Ces pratiques sont admissibles lorsque le consommateur en assume lui-même les frais. La ques pratiquant une pensée causale. scientifique. homöopathische Behandlung von Heufieber publiziert [11]. Die in einem «letter to the editor» von sechs namhaften Pharmakologen und Klinikern der Schweiz und der USA geäusserte Kritik (Untauglichkeit des multisymptomatischen Heufiebers für einen RCT mit dem Code ausschliesslich in den Händen eines homöopathischen Apothekers und der zusätzlichen Verabreichung von Antihistaminika) wurde vom Lancet nicht publiziert [7].

Keiner der zehn Befunde im Beitrag von Albonico und Ferroni $[1,2]$ kann die Entlarvung der Homöopathie als Placebo erschüttern. Mit der Definition der Homöopathie als «hochindividualisierte komplexe Therapiemethoden» wird versucht, den RCT als Methode darzustellen, die für die Wirksamkeitsprüfung der Homöopathie la vente de placébos nuisent à l'individu et à la collectivité. Le scepticisme envers d'anciennes pratiques insuffisamment ou pas du tout vérifiées, ainsi qu'envers des nouveautés recommandées prématurément, mérite d'être soutenu dans l'espoir qu'à plus ou moins court terme, on saura du moins en médecine, dépasser le stade des illusions, du moins en médecine, afin de suivre l'exemple des scientifi-

En ce qui concerne l'information sur les anciennes pratiques et les nouveautés en matière de thérapies, la plupart des médias ne s'intéressent pas à la vérité et s'adonnent aux illusions. II arrive que même des journalistes de revues réputées succombent à des tentatives de mystification se basant sur des pratiques trompeuses qui, à I'aide des politiques et des médias, parviennent à pénétrer dans les universités dans le but de se créer une crédibilité 
nicht geeignet sei. Bekanntlich handelt es sich bei der korrekten Durchführung des RCT um die objektivste Methode zur Prüfung der Wirksamkeit von medizinischen Massnahmen [12, 12a]. Der zitierte Entwurf des WHO-Berichtes, nach dem die Homöopathie in placebokontrollierten Studien dem Placebo überlegen und mit den konventionellen Therapien von Krankheiten von Mensch und Tier gleichwertig sei, ist von mehreren Experten (Edzard Ernst und dem Vorsitzenden der «Dutch Union against Quackery» Cees Renckens) als voreingenommen und «prohomoeopathy propaganda» kritisiert worden [10].

Die Homöopathen klammern sich noch an den Glauben, dass der fehlende wissenschaftliche Nachweis der Wirksamkeit eine Wirkung nicht ausschliesse [4].

\section{Die fehlende Wissenschaftlichkeit der Homöopathie}

In zwei Publikationen wurde darauf hingewiesen $[7,8]$, dass praktisch alle homöopathischen Forschungsinstitute, in denen ursprünglich überzeugte Homöopathen die Wissenschaftlichkeit beweisen sollten, nach einer gewissen Zeit die Homöopathie in Forschung und Lehre wieder aufgaben. Auch das Hahnemann Medical College in Philadelphia gab die Homöopathie in Forschung und Lehre auf und funktionierte weiter als moderne «Medical School». Entsprechend der «NZZ am Sonntag» vom 22. Juni 2003 behauptete der Lausanner Physiker L. Rey, dass sich Wassermoleküle an Substanzen erinnern, mit denen sie vor deren Elimination durch hochgradige Verdünnung in Kontakt waren. Die in der Zeitschrift «Physica A» publizierte Studie war gesponsert. Sie erinnert an den 1988 ebenfalls von der homöopathischen Industrie gesponserten Versuch, der Homöopathie ein wissenschaftliches Mäntelchen umzuhängen. Der in der renommierten Zeitschrift «Nature» erschienene Unsinn [13] wurde korrigiert [14], und die Richtigstellung erfolgte später nochmals durch kontrollierte Versuche im University College in London [15]. Damit war die Homöopathie definitiv als Irrlehre und als einer der grössten Irrtümer in der Geschichte der Heilkunde entlarvt. Der Fachbereich der Humanmedizin der Universität Marburg hat am 2. Dezember 1992 in der «Marburger Erklärung» die Homöopathie als Irrlehre verworfen und darauf hingewiesen, dass die beiden Prinzipien derselben - die Ähnlichkeitsregel und das Potenzieren durch Verdünnunga - dem vernünftigen Denken, dem die Universitäten verpflichtet sind, widersprechen, die Patienten täuschen und einem publizistisch geschürten Aberglauben entsprechen [7].
Unter allen Publikationen über die Homöopathie ragt die Monographie «Homöopathie - kritisch betrachtet» des 2006 verstorbenen Zürcher Pharma- und Toxikologen W. H. Hopff heraus, weil sie die Unvereinbarkeit der Homöopathie mit den bekannten Fakten der Chemie und Physik hieb- und stichfest offenlegt. Hopff hat in seiner umfassend dokumentierten Monographie, die nirgends, nicht einmal in der Literaturstudie des Lancet [3], zitiert wird, darauf hingewiesen, dass die Homöopathie im Anschluss an den 2 . Weltkrieg praktisch bedeutungslos wurde. Ihre Wiederbelebung erfolgte jedoch bald einmal mit Werbesprüchen wie «Wer heilt, hat recht» und Schlagworten wie «Ganzheitsmedizin» und «Alternativ- und Komplementärmedizin». «Placeboforscher» bemühen sich, mit Hilfe von bildgebenden Techniken Veränderungen im Gehirn nach Scheinbehandlungen darzustellen. Die psychischen Nebenwirkungen von Placebos mit Ausschüttung von Neurotransmittern und Hormonen ist von Hopff bereits 1991 als «Autopharmakologie» beschrieben worden [7]. Die Ausschüttung von schmerzlindernden Endorphinen (z.B. bei der Akupunktur) wird dazu verwendet, den zahlreichen Placebopraktiken der Alternativ- und Komplementärmedizin den Anschein von Wirksamkeit gegen Krankheiten zu verleihen.

\section{Die Popularisierung von heilerischem Leerlauf durch Politik und Medien}

$\mathrm{Zu}$ erwähnen sind das bei einem Einsatz von 6 Mio. Franken ohne Wirksamkeitsnachweis verlaufene Nationale Forschungsprogramm «NFP 34 Komplementärmedizin» von 1992 bis 1998 und das mit nochmals 6 Mio. Franken als Marktforschungsstudie betriebene «Programm Evaluation Komplementärmedizin PEK» [16], das ebenfalls inklusive die Literaturstudie keinen Wirksamkeitsnachweis brachte. Placebopraktiken werden nicht wirksam, wenn man sie immer wieder und sogar mit untauglichen Methoden testet und die negativen Ergebnisse nicht wahrhaben will.

Die Tatsache, dass die NZZ vom 19. November 2003 unter «Forschung und Technik» der Homöopathie eine ganze Seite samt dem Bild des Gründers widmete, zeigt die unglaubliche Überlebenskraft von heilkundlichen Trugschlüssen. Hahnemann wurde nach einer Überdosis von Chinin aus Chinarinde, das eine Untertemperatur erzeugt, von «Fiebern» geschüttelt. ${ }^{b}$ Dies führte ihn zur Idee, mit einer verschwindend kleinen Dosis Chinin den Körper an Fieber «gewöhnen» zu wollen. So entstand seine Theorie, Krankheiten könnten durch Substanzen behandelt werden, die bei Gesunden dieselben Symptome provozieren («Similia Similibus curentur»). In einem am 
4. Oktober 2006 in der NZZ unter dem Titel «Placebo - oder die Hoffnung heilt» publizierten Artikel wurde die despektierliche Ansicht geäussert, dass die Rolle von Schamanen und Geistheilern heute den Ärzten zufalle, die sich «gewissermassen als Placebo einbringen sollten». Die bereits erwähnten mit Hilfe von bildgebenden Techniken feststellbaren Veränderungen im menschlichen Gehirn als Nebenwirkung von Scheinmedikamenten und Scheinoperationen tragen nichts zum Verständnis von ernsthaften Krankheiten und ihrer Heilung oder Verhinderung bei.

Für die Propagierungsrolle der Medien zugunsten von irreführenden Scheinbehandlungen gibt es ein weiteres Beispiel in Form der Beeinflussung des Laienvolkes für die Akzeptanz von zwei Irrlehren aus dem 19. Jahrhundert, der Osteopathie und Chiropraktik. In zwei von namhaften Zürcher und Berner Klinikern verfassten Gutachten [18] wurden die Osteopathie und Chiropraktik als Irrlehren entlarvt. Die von Andrew Taylor Still 1876 erfundene Osteopathie und die vom Spezereihändler und Magnetheiler E. Palmer nachgeahmte Chiropraktik behaupteten, mit der manipulativen Behebung von «Fehlstellungen» der Wirbelgelenke verschiedenste von diesen «Fehlstellungen» verursachte Krankheiten heilen zu können. Diese Theorien widersprechen allen im 20. Jahrhundert erkannten anatomischen, physiologischen und pathologischen Fakten des menschlichen Organismus.

Da die Absurdität dieser Vorstellungen offensichtlich wurde, beschäftigt sich die ursprünglich als Heilsystem für die meisten Krankheiten verwendete Chiropraktik gemäss den Angaben des Instituts für Weiterbildung der Schweizerischen Chiropraktorengesellschaft nur noch mit «funktionellen, reversiblen Störungen des Bewegungsapparates, insbesondere der Wirbelsäule, des Nervensystems, der Muskeln, Sehnen und Gelenke». Das heutige kleinere Betätigungsfeld der Chiropraktik betrifft somit den Bewegungsapparat, für den die orthopädische Chirurgie zusammen mit der Neurologie, Neurochirurgie und der Rheumatologie auf solider wissenschaftlicher Basis zuständig und recht erfolgreich tätig ist. Die neuen Untersuchungen der komplexen Nervenversorgung der Gelenke, Ligamente und Muskeln der Wirbelsäule haben lediglich Spekulationen über den bei einer Manipulation in Gang gesetzten neurophysiologischen Vorgang gebracht. Weder die Chiropraktik noch die sogenannte manuelle Medizin haben bis heute den Beweis geliefert, ernsthafte, nicht spontan remittierende Affektionen des Bewegungsapparates beheben zu können und mehr zu leisten als alle übrigen Scheinbehandlungen der Alternativ- und Komplementärmedizin.

Die versierten Chiropraktoren erreichten die Anerkennung der Chiropraktik als dem Arzt-, Zahnarzt- und Apothekerberuf gleichgestellter Medizinalberuf und die Aufnahme in die solidarisch finanzierte Grundversicherung. Nach dem Studium der in Vergessenheit geratenen hiebund stichfesten Zürcher und Berner Gutachten über die Chiropraktik [18], nach der Kenntnisnahme von durch unnötige Manipulation der Halswirbelsäule entstandenen Gefässverletzungen und Lähmungen und nach der Erfahrung mit wiederholten Manipulationenc bin ich erstaunt über die Absichten des Zürcher Universitätsrates. Gemäss einer Mitteilung in der NZZ vom 25. Januar 2007 will dieser Rat (bestehend aus zwei Nationalrätinnen, einer Juristin, einem Mediziner, einem Wirtschaftswissenschafter, einem Maschineningenieur und einem Journalisten) eine Assistenzprofessur für Chiropraktik schaffen und mittelfristig einen Studiengang Chiropraktik an der medizinischen Fakultät der Universität Zürich einrichten.

\section{Hat die Vernunft im Gesundheitswesen des 21. Jahrhunderts eine Chance?}

Es ist selbstverständlich allen Menschen unbenommen, Scheinbehandlungen zur momentanen Beeinflussung des subjektiven Befindens auf eigene Verantwortung zu konsumieren und selbst zu finanzieren. Aber es ist erfreulich, dass es mutige Politiker und unabhängige scharfsinnige Redaktoren, Ärzte und Publizisten gibt, die sich für die Wahrheitssuche mit Hilfe des vernünftigen Denkens $[6,7,17,19-21,23]$ einsetzen, um das illusionäre Wunschdenken in die Schranken zu weisen. Wenn Massnahmen nicht nur der wissenschaftlich fundierten Medizin, sondern auch der sogenannten Alternativ- und Komplementärmedizin rigoros auf Wirksam- und Notwendigkeit geprüft und von der solidarisch finanzierten Krankenversicherung ausgeschlossen werden, wenn sie die Wirksamkeitsprüfung nicht bestehen, fallen lediglich vermögende Bürgerinnen und Bürger dem heilerischen Leerlauf zum Opfer. Die hartnäckige Unterstützung der Vernunft lässt hoffen, dass es vielleicht früher oder später gelingen wird, das 1919 vom scharfsinnigen Psychiater Eugen Bleuler beschriebene autistisch-undisziplinierte Denken, das als Wunsch- oder dogmatisches Denken in Politik, Religion und in Medien allzuhäufig schadet, wenigstens in der Heilkunde und im Gesundheitswesen zu überwinden $[6,17,20-23$, 25]. Zum mindesten sollte die Hoffnung nicht aufgegeben werden, dass das Ende von Irrlehren und Scheinbehandlungen im Laufe des 21. Jahr- sein Krankheitsgefühl und Behandlungsbedürfnis unterhalten. 
hunderts näherkommen wird. Wenn aufgeklärte, seriös informierte Bürgerinnen und Bürger künftig wegen eines lästigen, behindernden oder gar lebensbedrohenden Leidens einen Arzt aufsuchen, um ihn um Rat und Hilfe zu bitten, erwarten sie von ihm und seinen Kollegen eine wahrheitsgetreue Beurteilung und Informationen über die Erfolgsaussichten von verfügbaren, seriös geprüften Behandlungsmöglichkeiten und über die wahrscheinlichen Folgen beim Verzicht auf Behandlung.

Informierte Bürgerinnen und Bürger des 21. Jahrhunderts erwarten, dass die von ihnen frei gewählten Ärzte vernünftig erzogene, fachlich kompetente, vertrauenswürdige, zuverlässige und mitfühlende Mitmenschen sind, von denen sie nicht mit einer Scheinbehandlung «ut aliquid fieri videatur» hinters Licht geführt werden.

Verbesserungen der «condition humaine» entstanden und entstehen durch die Erkennung und Beseitigung von Dogmata, Irrtümern und Irrlehren und nicht durch die Pflege derselben während Jahrhunderten oder gar Jahrtausenden. Die erst im Laufe des 19. Jahrhunderts entstandene, somit noch junge naturwissenschaftlich fundierte Medizin erzielte in der kurzen Zeit von 150 Jahren endlich langersehnte Erfolge gegen Seuchen [22] und Krankheiten und bei der chirurgischen Reparation von Verletzungen und Krankheitsfolgen. Und sie steht nicht wie gerne behauptet am Ende, sondern mitten in ihren Bemühungen, die Spezies Homo sapiens von den noch unheilbaren, vor allem das Alter belästigenden Krankheiten zu befreien oder wenigstens ihre Folgen zu mildern. Es ist nicht klug, die verfügbaren Mittel mit der Behandlung von verwöhnten Gesunden zu verschwenden, die bereits im besten Alter durch voreilige und oberflächliche Informationen über Krankheiten und Risiken verängstigt werden, bis sie sich als scheinbar unheilbare Kranke mit grenzenlosen Behandlungsbedürfnissen verhalten [24], die zahlreiche verschiedenste Akteure im Gesundheitswesen gerne zu befriedigen versuchen [25].

\section{Zusammenfassung der Schlussfolgerung}

Der Beitrag erinnert an die gescheiterten Versuche, die Wirksamkeit und Wissenschaftlichkeit der Homöopathie zu beweisen. Die psychischen Nebenwirkungen von Placebos in Form einer kurzzeitigen, suggestiven Beeinflussung des subjektiven Befindens durch Ausschüttung verschiedener Neurotransmitter ändert nichts an der Tatsache der Wirkungslosigkeit von Placebos gegen ernsthafte Krankheits- und Verletzungsfolgen des menschlichen Organismus. Die Wahrheitssuche und damit der Fortschritt in der Heilkunde beruht wie auf allen Gebieten menschlicher Aktivität auf der Erkennung und Beseitigung von Irrtümern und Dogmata. Praktiken, die ihr Überleben der Selbstheilungs- und Regenerationskraft des menschlichen Organismus und der kurzzeitigen Beeinflussung des subjektiven Befindens bei Leichtgläubigkeit und Verzweiflung verdanken, verbessern die Wirksamkeit und Ökonomie des Gesundheitswesens nicht. Sie sind tolerabel, wenn der Konsument die Kosten selbst trägt. Toleranz dem Unsinn gegenüber und der Verkauf von Placebos schaden dem Individuum und der Allgemeinheit. Die Skepsis gegen nicht oder ungenügend geprüfte Antiquitäten und voreilig empfohlene Novitäten braucht Unterstützung in der Hoffnung, dass früher oder später die Überwindung des illusionären Denkens wenigstens in der Heilkunde mit den kausal verstehend denkenden Naturwissenschaftern als Vorbilder gelingt.

Bei der Information über heilkundliche Antiquitäten und Novitäten sind die meisten Medien an der Wahrheit nicht interessiert und frönen dem illusionären Denken. Selbst Redaktoren von renommierten Fachzeitschriften können Täuschungsversuchen erliegen, die von irreführenden Praktiken ausgehen, die mit Hilfe von Politik und Medien in die Universität drängen, um sich eine wissenschaftliche Glaubwürdigkeit zu verschaffen.

\section{Literatur}

1 Albonico H, Ferroni B. Die Homöopathiestudie: Anatomie einer statistischen Operation. Schweiz Ärztezeitung. 2006;87(28):1276-9.

2 Albonico H, Ferroni B. Etude sur l'homéopathie: Anatomie d'une operation statistique. Bull Méd Suisses. 2006;87(45):1954-7.

3 Shang A, Huwiler K, Nartey L, Jüni P, Dörig S, Sterne JAC, Pewsner D, Egger M. Are the clinical effects of homoeopathy placebo effects? Comparative study of placebo-controlled trials of homoeopathy and allopathy. Lancet. 2005;366:726-32.

4 Altman DG, Bland JM: Absence of evidence is not evidence of absence. BMJ. 1999;311:485.

5 The end of homoeopathy. Lancet. 2005;366:690.

6 Skrabanek P, Mc Cormick J. Follies and Fallacies in Medicine. Glasgow: The Terragone Press; 1989.

7 Hopff W. Homöopathie kritisch betrachtet. Stuttgart: Thieme; 1991.

8 Prokop O, Hopff W. Gibt es heute noch Schildbürgerstreiche? Schweiz Med Wochenschr. 1992; 122(46):1770-1.

9 Vandenbrouck JP. Homoeopathy and «the growth of truth». Lancet. 2005;366:691-2.

10 McCarthy M. Critics slam draft WHO report on homoeopathy. Lancet. 2005;366:705-6. 
11 Reilly DT, McSharry C, Taylor MA, Aitchison T. Is homoeopathy a placebo response? Controlled trial of homoeopathy potency, with pollen in hayfever as model. Lancet. 1986;II:881-5.

12 Ernst E. What's the point of rigorous research on complementary/alternative medicine. J R Soc Med. 2002;95:211-3

12a Geiser M. 50 Jahre Randomised clinical control trials. Schweiz Ärztezeitung. 2003;84(13):598-601.

13 Davenas E, et al. Human basophil degranulation triggered by very dilute antiserum against JgE. Nature. 1988;333:816-8.

14 Maddox J, Randi J, Steward WH. High dilution experiments a delusion. Nature. 1988;334:287-90.

15 Hirst SJ, Hayes NA, Burridge J, Pearce FL, Foreman JC. Human basophil degranulation not triggered by very dilute antiserum against JgE. Nature. 1993; 366:525-7.

16 Geiser M. Das Ansehen nicht nur der FMH steht auf dem Spiel. Schweiz Ärztezeitung. 2005; 86(8):470-2.

17 Bleuler E. Das autistisch-undisziplinierte Denken in der Medizin und seine Überwindung. Berlin: Springer; 1927.
18 Geiser M. Die vergessenen Gutachten über die Chiropraktik. Schweiz Rundschau Med (Praxis). 1993;82:875-9.

19 Biedenkopf K. Die Ausbeutung der Enkel. Plädoyer für die Rückkehr zur Vernunft. Berlin: Propyläen; 2006.

20 Cochrane AL. Effectiveness and Efficiency. Random Reflections on Health Services. The Nuffield Provincial Hospitals Trust. Abindon: Burges and Son; 1972.

21 Abgrall JM. Les charlatans de la santé. Paris: Documents Payot; 1998

22 Winkle S. Geisseln der Menschheit. Kulturgeschichte der Seuchen. 3. Auflage. Frankfurt: Artemis und Winkler; 2005.

23 Thomas L. Medical lessons from History in «The Medusa and the Snail». New York: The Viking Press; 1979.

24 Klaesi J. Der unheilbar Kranke und seine Behandlung. Rektoratsrede. Bern: Paul Haupt; 1950.

25 Neftel K. «Disease mongering» und Skrabaneks «septicemia». Schweiz Med Forum. 2006; 6(46):1021-2 